\title{
Observing dynamical SUSY breaking with lattice simulation
}

\author{
Issaku Kanamori
}

Theoretical Physics Laboratory, RIKEN, Wako, Saitama 351-0198, JAPAN

\begin{abstract}
On the basis of the recently developed lattice formulation of supersymmetric theories which keeps a part of the supersymmetry, we propose a method of observing dynamical SUSY breaking with lattice simulation. We use Hamiltonian as an order parameter and measure the ground state energy as a zero temperature limit of the finite temperature simulation. Our method provides a way of obtaining a physical result from the lattice simulation for supersymmetric theories.
\end{abstract}

Keywords: supersymmetry, lattice, dynamical breaking PACS: $11.15 . \mathrm{Ha}, 11.30 . \mathrm{Pb}, 11.30 . \mathrm{Qc}$

\section{INTRODUCTION}

In these several years, lattice formulation of supersymmetric theories made a great progress [1, 2, 3, 4, 5, 6] (for the review, see [7]). Not only the formulations but the simulations have been already started [8, 9, 10].

We have developed a method of observing dynamical SUSY breaking using a lattice simulation [11, 12]. Since the SUSY is not broken in the perturbation if it is not broken in the tree level, a way of observing SUSY breaking due to non-perturbative effects is very important. Usually, the Witten index provides such a method. But sometimes it is not available. In fact the main target in this talk, two-dimensional $\mathscr{N}=(2,2)$ super YangMills theory (SYM) is such an example. What we know is only a conjecture which states it may be broken [13].

Our method uses the Hamiltonian as an order parameter. The requirement for the lattice model is an exact $Q$ symmetry on the lattice so it can be widely used in principle. After a very quick explanation of putting SUSY on the lattice using Sugino model [2], we state our method of measuring the Hamiltonian. After confirming that the method actually works, we will show the result for the super Yang-Mills case.

\section{LATTICE MODEL}

We use a lattice model with one exactly kept supercharge. It seems impossible to put the SUSY on the lattice, because SUSY algebra contains infinitesimal translation but on the lattice we have only finite translations. However, what we have realized in the recent development is that it is possible if $\mathscr{N} \geq 2$, especially in lowdimensional case. Most of the recent development utilize the topological twist. After the twist, we have a scalar supercharge instead of spinors. We can put the scalar on a lattice site and keep it exact at finite lattice spacing. Some of the simulation have already done aiming the check of the formulation.

We adopt such a model proposed by Sugino [2]. The target theory in the continuum has twisted $\mathscr{N}=2$ supersymmetry. It has the following algebra:

$$
\begin{aligned}
Q^{2} & =\delta_{\phi}^{\text {(gauge })}, \quad Q_{0}^{2}=\delta_{\bar{\phi}}^{\text {(gauge })}, \\
\left\{Q, Q_{0}\right\} & =2 i \partial_{0}+2 \delta_{A_{0}}^{\text {(gauge })}
\end{aligned}
$$

where we pick up only a part of the whole algebra. $\delta_{\bullet}^{\text {(gauge) }}$ denotes infinitesimal gauge transformation with the parameter $\bullet$. After the twist, we have four supercharges, a scalar $Q$, two from a two-dimensional vector $Q_{\mu}(\mu=0,1)$, and a pseudo scalar $\tilde{Q}$. They are nilpotent up to gauge transformation. The field components are a complex scalar field $\phi\left(\bar{\phi}=\phi^{\dagger}\right)$, gauge field $A_{\mu}$, Majorana fermions in the twisted basis $\eta, \chi$ and $\psi_{\mu}$, and an auxiliary field $H$.

The point is that the action has $Q$-exact form $S=$ $Q \Lambda$, where $\Lambda$ is a gauge invariant quantity, and $Q$ can be discretized keeping the nilpotency even at a finite lattice spacing. Therefore, the lattice action defined as $Q$-exact quantity is manifestly $Q$-invariant and enjoys $Q$ symmetry at a finite lattice spacing as long as it is gauge invariant. The lattice version of the $Q$ transformation are the following:

$$
\begin{array}{rlrl}
Q U(x, \mu)= & i \psi_{\mu}(x) U(x, \mu), & & \\
Q \psi_{\mu}(x)= & i \psi_{\mu}(x) \psi_{\mu} & & \\
& -i\left(\phi(x)-U(x, \mu) \phi(x+\hat{\mu}) U(x, \mu)^{-1}\right), \\
Q \phi(x)= & 0, & & \\
Q \chi(x)= & H(X), & & Q H(x)=[\phi(x), \chi(x)], \\
Q \bar{\phi}(x)= & \eta(x), & Q \eta(x), \bar{\phi}(x)],
\end{array}
$$

where $U(x, \mu)$ is a gauge link variable.

Eventually, we have nilpotent $Q$ and $Q$-invariant action. The remaining $Q_{0}, Q_{1}$ and $\tilde{Q}$ will be automatically restored in the continuum limit. 


\section{METHOD}

We use Hamiltonian as the order parameter since it is zero if and only if the SUSY is not broken. Therefore, it is very sensitive about the choice of the origin of the Hamiltonian. We use the SUSY algebra to define the Hamiltonian. From the algebra (2), we have

$$
Q \mathscr{J}_{0}^{(0)}=2 \mathscr{H}
$$

where $\mathscr{J}_{0}^{(0)}$ is the 0 -th component of the Noether current for $Q_{0}$ and $\mathscr{H}$ is a Hamiltonian density. On the lattice we have only $Q$ transformation but no $Q_{0}$ transformation. Therefore, we discretize the continuum version of the Noether current by hand. We know the $Q$ transformation so it is straightforward to obtain the Hamiltonian.

An advantage of measuring the Hamiltonian is that it is a one-point function. Compared with two-point functions which are used for measuring spectrum, it is numerically very cheap and easy to calculate.

Another point of our method is the boundary condition. In the lattice simulation, the lattice size is limited and usually we use a periodic condition. For the current purpose, however, we need a different choice.

As usual method for observing spontaneous symmetry breaking, we apply an external field conjugate to the order parameter. The conjugate to the Hamiltonian is the temperature. That is, we impose the anti-periodic condition in the time direction for fermion. Therefore we break SUSY by boundary condition or equivalently by the temperature. Then we take zero temperature limit and observe the effect of the breaking is left or not.

It is interesting to see what would happen if we took the periodic conditions. Under the periodic condition, the simulation does not work. Since the periodic partition function is exactly the Witten index, it is easy to see the expectation value of the Hamiltonian is proportional to the derivative of the Witten index $\langle\exp (-\beta H)\rangle_{\mathrm{PBC}}$ :

$$
\langle H\rangle_{\mathrm{PBC}}=\frac{-\frac{\partial}{\partial \beta}\langle\exp (-\beta H)\rangle_{\mathrm{PBC}}}{\langle\exp (-\beta H)\rangle_{\mathrm{PBC}}} .
$$

Because the index does not depend on the coupling $\beta$ 1, the derivative w.r.t. $\beta$ is zero so the expectation value of the Hamiltonian seems zero as well. If the SUSY is broken, however, the denominator is also zero and the expectation value of the Hamiltonian can be nonzero value. In the simulation, what we measure is the numerator. The path integral is replace by an ensemble average and the normalization factor is replaced by the size of the ensemble. Therefore, the simulation should

\footnotetext{
1 In the anti-periodic case, $\beta$ can be understood as the inverse temperature.
}

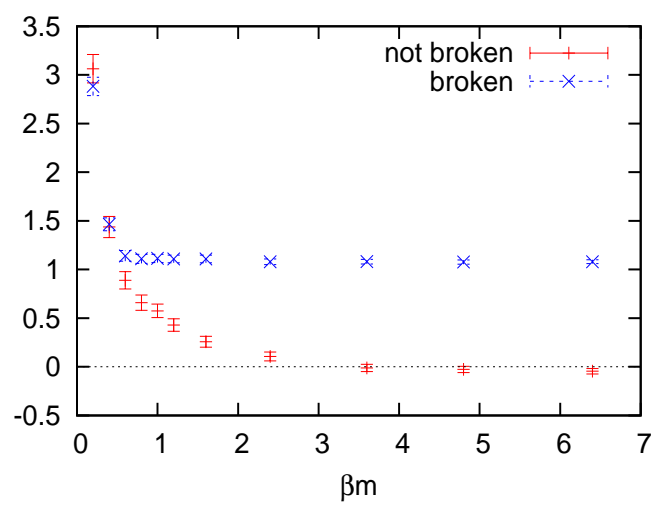

FIGURE 1. Hamiltonian of SQM versus inverse temperature $\beta$, in unit a dimensionful parameter $m$ in the potential. The plot is after taking the continuum limit. The "not broken" data goes to zero as the inverse temperature $\beta$ goes large, while the "broken" data stays finite. We can easily distinguish these two cases so our method actually works.

give always zero even if the SUSY is broken under the periodic boundary condition.

In fact the expectation value of our $Q$-exact Hamiltonian is always zero under the periodic boundary condition, if we ignore the possible zero of the denominator. Since the action is $Q$-invariant and the $Q$ is nilpotent, the expectation value of $Q$-exact quantity is zero. This is consistent with the above argument.

Eventually, we should measure the ground state energy as a zero temperature limit of the $Q$-exact Hamiltonian.

\section{RESULT}

As a check of our method, we first investigate Supersymmetric Quantum Mechanics (SQM). The known fact is that the form of the potential decides whether SUSY is broken or not. The lattice model we use was given in [14] and keeps nilpotent $Q$ and $Q$-exact action. The figure 1 shows that our method actually works. Next let us investigate the SYM case. See figure 2] We plot results obtained by two different simulation algorithms, new one and old one presented in [11, 12]. The errors are drastically reduced in the new plot and the ground state energy seems small 2 Because of the $Q$-exactness, the periodic Hamiltonian should be zero. In fact it is consistent with zero within the error (figure 3) and consistent with the discussion based on the Witten index.

2 Before giving conclusive statement on the SUSY breaking, we should confirm that the lattice model we use actually describes the target theory in the continuum [15]. 




FIGURE 2. Hamiltonian of SYM versus inverse temperature $\beta$, in unit of the dimensionful coupling $g$. The old data was obtained by reweighting method and the new data was obtained by RHMC method. The both indicate small ground state energy.

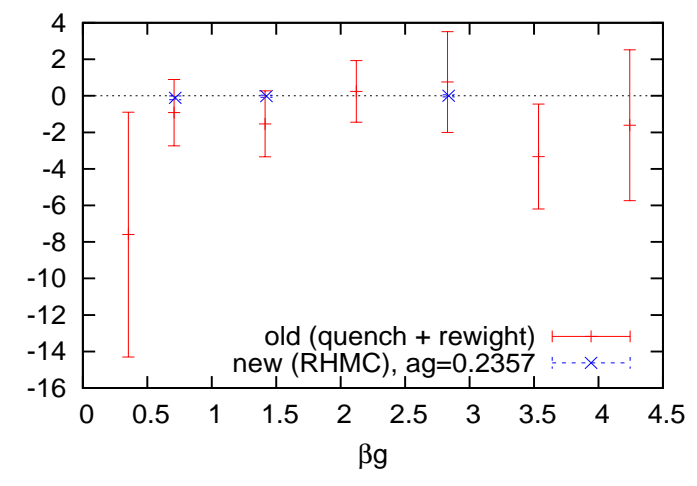

FIGURE 3. Hamiltonian versus inverse temperature $\beta$, under the periodic boundary condition. In the reweighting method the continuum limit is taken while in the RHMC method the lattice spacing is fixed at $a g=0.2357$. The values should be zero even at the finite lattice spacing.

Some details of the simulation of SYM are in order. The fermion effect is treated by a reweighting method or Rational Hybrid Monte Carlo (RHMC). The reweighting method uses quenched configurations and treats the fermion effect as a part of the observable so it is some how indirect. The RHMC method uses configurations with fermion effects. The simulation codes are developed based on the FermiQCD [16] and parameters for rational approximation in the RHMC are obtained from [17]. We fix the physical spacial size to 1.41 in unit of dimensionful gauge coupling $g$ 百 The parameters are the following. The lattice size is $3 \times 6-36 \times 12$ and the lattice spacing is $0.0707-0.2357$. The number of the independent configurations are 9900-99900 in the reweighting method and $10-1700$ in the RHMC method.

\footnotetext{
${ }^{3}$ All the dimensionful quantities are measured in unit of $g$.
}

\section{CONCLUSION}

We have developed a method of observing dynamical SUSY breaking using lattice simulation. Our method is available if the lattice model has one exactly kept supercharge $Q$ and $Q$-exact action. We used Hamiltonian as the order parameter and measure the ground state energy. It actually worked in supersymmetric quantum mechanics, a system whether SUSY is broken or not was already known. Then we applied it to two-dimensional super Yang-Mills. It is the first physical application of the recent development of the lattice SUSY. The application to the other system is straightforward. Now we can use the lattice formulation for supersymmetric theories.

\section{ACKNOWLEDGMENTS}

The author would like to thank M. Hanada, H. Kawai, H. Matsufuru, K. Murakami, F. Sugino and H. Suzuki. He is supported by the Special Postdoctoral Researchers Program at RIKEN. The simulation result of super YangMill model was obtained by using Riken Super Combined Cluster System (RSCC).

\section{REFERENCES}

1. D. B. Kaplan, E. Katz and M. Unsal, JHEP 0305037 (2003) |hep-lat/0206019|.

2. F. Sugino, JHEP 0401015 (2004) [hep-lat/0311021].

3. S. Catterall, JHEP 0411006 (2004) |hep-lat/0410052|.

4. A. D'Adda, I. Kanamori, N. Kawamoto and K. Nagata, Nucl. Phys. B 707100 (2005) [hep-lat/0406029].

5. H. Suzuki and Y. Taniguchi, JHEP 0510082 (2005) |hep-lat/0507019|.

6. J. W. Elliott and G. D. Moore, PoS LAT2005 245 (2006) [JHEP 0511010 (2005)] |hep-lat/0509032].

7. J. Giedt, PoS LAT2006 008 (2006) |hep-lat/0701006|.

8. S. Catterall, JHEP 0603032 (2006) |hep-lat/0602004|.

9. H. Suzuki, JHEP 0709052 (2007) [arXiv:0706.1392].

10. H. Fukaya, I. Kanamori, H. Suzuki and T. Takimi, PoS LAT2007 264 (2007) |arXiv:0709.4076].

11. I. Kanamori, H. Suzuki and F. Sugino, Phys. Rev. D 77 091502 (2008) |arXiv:0711.2099|.

12. I. Kanamori, F. Sugino and H. Suzuki, Prog. Theor. Phys. 119797 (2008) |arXiv:0711.2132.

13. K. Hori and D. Tong, JHEP 0705079 (2007) |hep-th/0609032].

14. S. Catterall and E. Gregory, Phys. Lett. B 487349 (2000) |hep-lat/0006013|.

15. I. Kanamori and H. Suzuki, in preparation.

16. M. Di Pierro, Comput. Phys. Commun. 14198 (2001) |hep-lat/0004007|.

17. M.A. Clark and A.D. Kennedy, http://www.ph.ed.ac.uk/mike/remez mike/remez, 2005. 